\title{
Activation of Graphenic Carbon due to Substitutional Doping by Nitrogen: Mechanistic Understanding from First-principles
}

\author{
Joydeep Bhattacharjee \\ School of Physical Sciences \\ National Institute of Science Education and Research \\ IOP campus, Sachivalay Marg, Bhubaneswar-751005, India
}

September 16, 2021

\begin{abstract}
Nitrogen doped graphene and carbon nanotubes are popularly in focus as metal-free electro-catalysts for oxygen reduction reactions (ORR) central to fuel-cells. $\mathrm{N}$ doped CNTs have been also reported to chemisorb mutually, promising a route to their robust pre-determined assembly into devices and mechanical reinforcements. We propose from first-principles a common mechanistic understanding of these two aspects pointing further to a generic chemical activation of carbon atoms due to substitution by nitrogen in experimentally observed configurations. Wannier-function based orbital resolved study of mechanisms suggests increase in C-N bondorders in attempt to retain $\pi$-conjugation among carbon atoms, causing mechanical stress and loss of charge neutrality of nitrogen and carbon atoms, which remedially facilitate chemical activation of $\mathrm{N}$ coordinated $\mathrm{C}$ atoms, enhancing sharply with increasing coordination to $\mathrm{N}$ and proximity to zigzag edges. Activated $\mathrm{C}$ atoms facilitate covalent adsorption of radicals in general, diradicals like $\mathrm{O}_{2}$ relevant to ORR, and also other similarly activated $\mathrm{C}$ atoms leading to self-assembly of graphenic nano-structures, while remaining inert to ordinary graphenic C atoms.
\end{abstract}

Disruption of $\pi$-conjugation due to substitution by $p-$ or $n$ - type dopants in graphene causes localization of $2 p_{z}$ electrons, which are a rich source of exotic physical as well as chemical functionalities unavialable in undoped graphene. Accordingly, boron (B) and nitrogen (N) doped graphene (GF) and carbon nanotubes(CNT) have been under rigorous scrutiny during the last decade or so, resulting into a great multitude of proposals for novel applications ranging from nano-electronics to catalysis. Electro-catalytic functionality of doped GFs and CNTs have been under investigation (1, 2, 3) for more than a decade, primarily in pursuit of an efficient metal-free catalyst to replace the expensive platinum based ones used in fuel-cells so far. In particular, N doped CNTs and GFs(4, 5, 6, 17, 8, 9, 10, 11, 12), and more recently their composites(13, 14, 15, 16, 17), have been explored exhaustively in the last few years as catalysts for oxygen reduction reactions (ORR) promisimg removal of platinum from the cathode in fuel-cells. On the other hand, $\mathrm{N}$ doping has also been suggested(18) to induce covalent mutual adsorption among CNTs through C-C cross-links, as has indeed been reported(19), promising a new route to their controlled assembly into devices and stronger composites, which in itself is an important open problem. In the present work we show mechanistically that indeed these two aspects are just different facets of a more inclusive general scenario of chemical activation of $\mathrm{C}$ atoms arising with experimentally observed configurations of substitutional doping by $\mathrm{N}$ in $s p^{2}$ hybridized carbon (C) based graphenic nano-structures (GN).

First principles studies(20, 21, 22, 23, 24) of ORR catalysis so far, primarily of isolated mono-substitution by N, suggest adsorption of atomic, molecular as well as active anionic oxygen $(\mathrm{O})$ on $\mathrm{C}$ atoms in the vicinity of quaternary $\mathrm{N}$ atoms, presumably driven by the effective positive charge of $\mathrm{C}$ atoms due to electron extraction by their $\mathrm{N}$ neighbours, which however does not explain the observed mutual adsorption of $\mathrm{N}$ doped CNTs. Interestingly, recent direct observations (25, 26) suggest substitution by $\mathrm{N}$ in GF to occur predominantly at next-nearest neighbouring (N-Nn) sites, which hinders one of the three degenerate $\pi$-conjugation configurations more than the other two, unlike in case of sparse mono-substitution where all three of them are equally hindered. This implored us to ask whether 


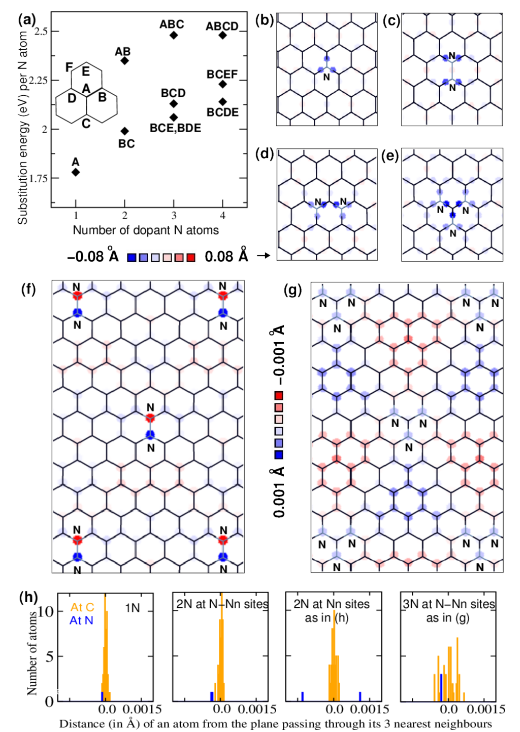

Figure 1: (a) Energetics of substitutional doping. (b-e) Variation in bond lengths compared to corresponding equilibrium values. (f,g) Contour plot of the degree of non-co-planarity measured as the vertical distance of an atom from the plane passing through its nearest neighbours. (h) Histogram of the degree of non-co-planarity for different substitution configurations.

multiple substitution at N-Nn sites can open up a new class of mechanisms supporting ORR catalysis as well as GN-GN covalent adsorption on the same footing. Additionally, as the N-Ns sites belong to one of the two sublattices of graphene, which support inter-sub-lattice spin-separation upon physical or chemical disparity between the two sub-lattices, the resultant magnetism needs to be linked to other functionalities arising out of substitution at N-Nn sites. Notably, inter-sublattice spin-separation, which manifests itself as the nearest neighbour anti-ferromagnetic order, and can be rationalized as an effect of on-site Coulomb repulsion between electrons with opposite spins, is naturally expected to contest $\pi$-conjugation, although modestly. Disparity between sub-lattices due to zigzag edges, which is well known to consolidate nearest neighbour anti-ferro-magnetism in GF, is thus expected to considerably influence properties of GNs with substitution by $\mathrm{N}$ at N-Nn sites.

Through unambiguous quantitative estimates of spin resolved bond orders and atomic charges obtained based on spatially localized Wannier functions(WF), we find the onset of mechanical strain and loss of charge-neutrality of $\mathrm{N}$ and $\mathrm{C}$ atoms due to evolution of the $\mathrm{C}-\mathrm{N}$ bond-orders in attempt to maximally retain $\pi$-conjugation among $\mathrm{C}$ atoms upon substitution by $\mathrm{N}$, to not only govern the energetics of substitution but also to favour passivation of $\mathrm{N}$ coordinated $\mathrm{C}$ atoms as an overall remedy. Such activated $\mathrm{C}$ atoms facilitate covalent adsorption of radicals, di-radicals like $\mathrm{O}_{2}$, as well as similarly activated $\mathrm{C}$ atoms leading to cross-linking of $\mathrm{N}$ doped GNs, although remain inert to ordinary graphenic $\mathrm{C}$ atoms, which is advantageous since it would allow spatially resolved self-assembly of $\mathrm{N}$ doped GNs. Adsorption of $\mathrm{O}_{2}$ as diradical leaves the mono-coordinated $\mathrm{O}$ atom charged and reactive as required for ORR. Activation is found to increase sharply with increasing coordination to $\mathrm{N}$ and proximity to zigzag edges. Absolute magnetization is observed to alter significantly in events of adsorption, since it restores $\pi$-conjugation to a variable degree, which in turn modifies nearest neighbour anti-ferromagnetism, given the anti-correlation between the two. In addition to proposing a new paradigm of activation of graphenic $\mathrm{C}$, this work also highlights the effectiveness of WFs in shaping precise orbital resolved understanding of mechanisms of chemical processes.

Equilibrium configurations and relevant energetics of substitutional doping are obtained within the non-empirical framework of density functional theory (DFT) based on local mean-field approximation of the many-electron exchange-correlation contributions to total energy. We use the Quantum Espresso(27) code, which expands wavefunctions in the basis of plane waves and allow ultrasoft(29) pseudopotentials for valence electrons. For exchange- 
correlation we use a gradient corrected Perdew-Burke-Ernzerhof (PBE)(28) functional. We considered a $5 \times 5$ GF super-cell to understand the energetics of substitution by $\mathrm{N}$ and the proposed activation of $\mathrm{C}$ atoms thereby. To study the effect of zigzag edges and use an available scheme for unbiased partitioning of total charge density in isolated systems, we also consider an isolated GF segment(Fig 4 (a)) made of 84 atoms. Total energies and configurations are converged with plane-wave cutoffs over $800 \mathrm{eV}, k$-mesh up to $7 \times 7 \times 1$ for the super-cell, and forces less than $10^{-4}$ Rydberg/Bohr using the Broyden-Fletcher-Goldfarb-Shanno (BFGS)(30) scheme for total energy minimization. To account for the attractive dispersion interactions, semi-empirical Grimme approximation(31) is used only in cases where chemisorption is not conclusive with PBE.

Unambiguous estimates of bond-orders, atomic charges and sub-shell filling, are obtained through construction of localized WFs based on joint-diagonalization of non-commuting first moment matrices (FMM). In one dimension, WFs with maximum localization(34) are naturally the eigen-functions of the FMM which is essentially the position operator expanded in the occupied subspace. For periodic systems the FMM is obtained in terms of geometric phases of Bloch states evolving over the full Brillouin zone. For isolated systems the FMMs can be directly calculated from the occupied Kohn-Sham (KS) eigen-states $\left\{\phi_{m}^{K S}\right\}$ as

$$
X_{m n}=\left\langle\phi_{m}^{K S}|\hat{x}| \phi_{n}^{K S}\right\rangle,
$$

for occupied KS states in the valence band. Diagonalizing a FMM (say $X$ as evaluated above) yields WFs with maximum localization in the corresponding $(x)$ direction. However, owing to the inherent non-commuting nature of the position operators expanded within the finite occupied subspace, it is not possible to obtain WFs with maximum localization simultaneously in all three directions. Nevertheless, approximate joint diagonalization of the three FMMs renders a set of highly localized WFs, which, even though strictly not unique, constitute an unambiguous orbital representation of the occupied sub-space. Their non-uniqueness arise only from the sequence in which the three FMMs are considered in course of the iterative approximate joint diagonalization process, and in effect has negligible bearing on the WFs rendered. Wannier centres (WCs), which are centre of masses of WFs, are readily available as the approximate eigen-values of the three FMMs, without having to explicitly construct the WFs. WCs, each representing one electron for each spin, provide a unique dot structure map for valence electrons through out the system. In case of partially occupied bands, the map is obtained by weighted sum of WCs for different number of KS states:

$$
W C(\vec{r})=\sum_{N=1}^{\infty}\left[\sum_{i=1}^{N} \delta\left(\vec{\gamma}_{i}-\vec{r}\right)\right]\left(f_{N+1}-f_{N}\right)
$$

where $f_{N}$ is the occupancy of the $N$-th KS state, such that

$$
\int_{-i n f t y}^{\infty} W C(\vec{r}) d \vec{r}=N_{e}
$$

where $N_{e}$ is the total number of valence electrons in the isolated system.

WCs can be identified in two categories - (1) associated with atoms, and (2) representing bonds. Single and double bonds are represented respectively by one and two WCs between two atoms. For visualization purpose, WCs in close proximity, like the ones representing double bonds, have been fused into one $\mathrm{WC}$ representing two electrons. WCs are represented by spheres, whose radii are scaled linearly by the number of electrons they represent, which is thus between 0 to 2. The depth of shading(in gray-scale) of the planar projection of these spheres are also linearly scaled in the same range. Number of electrons associated with an atom is implied by the WCs exclusively associated with it, representing unpaired and lone-paired electron(s) if any, and half the order of all the bonds made by the atom according to the WCs representing covalent bonds. WFs and WCs are constructed using an in-house implementation which takes the KS eigen states as input. The reason to use this method is that it does not depend on any reference template of orbitals, which is required for construction of localised WFs(35, 36) in periodic systems, where it also biases the resultant WFs towards the set of orbitals specified in the template. However, 


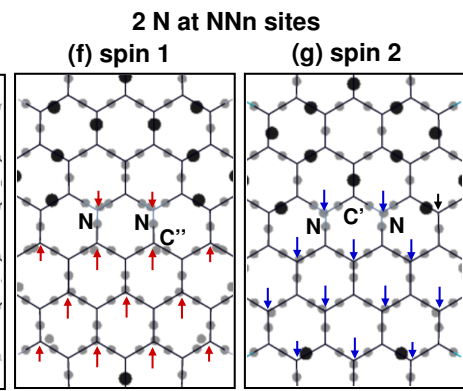

(I) Spin density with $3 \mathrm{~N}$ at $\mathrm{NN}$ sites $3 \mathrm{~N}$ at $\mathrm{NNn}$ sites (h) spin 1

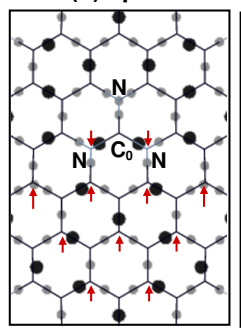
(i) spin 2

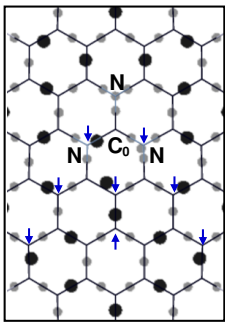

$\mathrm{CH}_{3}$ adsorbed on $\mathrm{C}_{0}$

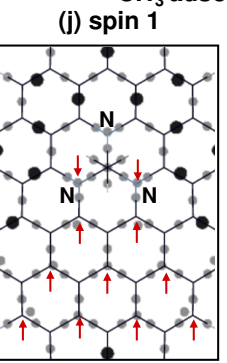

(k) spin 2

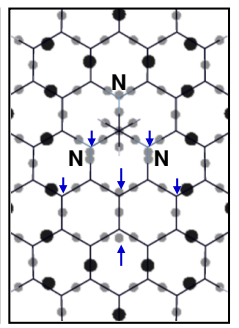

in $5 \times 5$ graphene supercell

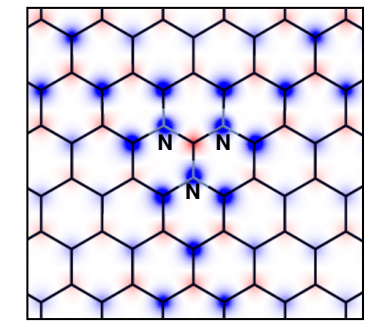

Figure 2: (a-k) Planar projection of WCs implying order of bonds, charge state, spin polarization and subshellfilling of atoms. Adjacent WCs have been merged into one WC located at their center of mass. Larger and darker circles each denote 2 electrons per spin, while the smaller gray circles represent 1 electron per spin. (l) Spin density $\left(\rho_{\text {spin } 1}-\rho_{\text {spin } 2}\right)$ with substitution at three mutually N-Nn sites enclosing a carbon atom.

as the method can not be directly extended to periodic systems, estimation of bond-orders and atomic charges are made based on WCs calculated in hydrogen passivated isolated GF segments, and the implied mechanisms are argued to be plausible in their corresponding periodic super-cells based on the similarities in bond-lengths, bond-angles and the degrees of non-co-planarity of atoms observed in the periodic super-cells and their isolated GF counterparts.

Energy required for substitution by $\mathrm{N}$ in the periodic super-cell, calculated as $\left(E_{\text {doped }}-E_{\text {undopped }}\right)+m E_{C}-$ $m E_{N}$ ), where $E$ is total energy and $m$ is the number of $\mathrm{C}$ atoms substituted, is plotted in Fig.1(a). Consistent with experimental observations (25, 26), Fig.1(a) implies substitution at nearest neighbouring (Nn) sites to be particularly unfavourable. The deviation in equilibrium bond-lengths plotted in Fig 1 (b-e) from that of the resonating graphenic C-C bonds and single (order 1) C-N and N-N bonds obtained with same parameters, suggests strain in the C-N bonds to be possibly responsible for difference in energetics of substitution. To translate these deviations into strain, bond orders have to be estimated, since reduction in length of a bond can also happen with increase in its order.Fig 2 (e) suggests complete retention of $\pi$-conjugation among the $\mathrm{C}$ atoms with substitution at Nn sites supporting C-N and N-N bonds or order 1, as readily understood by considering substitution at the two sites enclosed by the dashed rectangle in Fig 2 (a). The $\mathrm{C}$ atoms would thus strongly favour co-planarity among themselves, while the $\mathrm{N}$ atoms, owing to their lone pairs, would strongly favour non-co-planarity with their nearest neighbours. We indeed observed this in the periodic super-cell, as evident in Fig 1 (f), which maps the degree of non-coplanarity of an atom as its vertical distance from the plane passing through its three nearest neighbours. Thus with substitution at Nn sites, the C-N bonds in the periodic supercell are also likely to be of order 1, implying that those C-N bonds are indeed longitudinally strained as their observed reduction in length is not due to any increase in their order. The stark contrast between $\mathrm{C}$ and $\mathrm{N}$ atoms in their preference for non-coplanarity with their nearest neighbours also suggests non-nominal dihedral strain.

With substitution at non-Nn sites, completely unaltered retention of $\pi$-conjugation is impossible, resulting into ease in accommodation of $\mathrm{C}-\mathrm{N}$ single bonds, which are typically about $4 \%$ longer than the graphenic C-C bonds. 


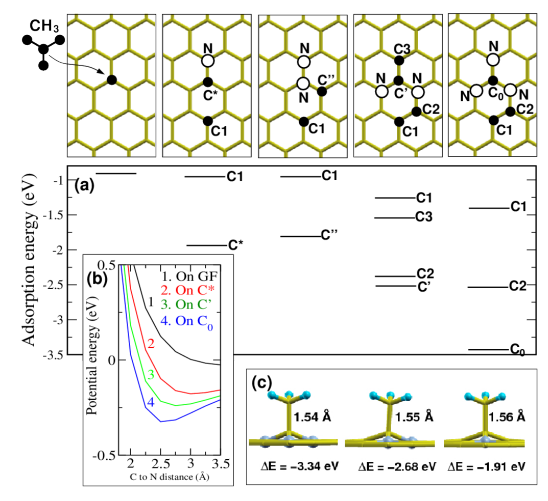

Figure 3: (a) Energetics of adsorption of $\mathrm{CH}_{3}$ on different $\mathrm{C}$ atoms. (b) Interaction potential of $\mathrm{CH}_{3}$ as a function of vertical distance of its $\mathrm{C}$ atom from the $\mathrm{C}$ atoms in periodic $\mathrm{GF}$ supercells specified in the figure. (c) Equilibrium configurations of adsorbed $\mathrm{CH}_{3}$ on $\mathrm{C}_{0}, \mathrm{C}^{\prime}$ and $\mathrm{C}^{*}$ in periodic $\mathrm{GF}$ supercell.

This is evident in Fig.1(d) which shows only two of the six C-N bonds to have significant reduction in length upon bi-substitution at N-Nn sites. Furthermore, Fig 22(c,h,i) suggests increase in order of C-N bonds with substitution at non-Nn sites, despite the consequent loss of charge neutrality of $\mathrm{N}$ atoms due to depletion from their lone pairs. This in effect imply that retention of $\pi$-conjugation among $\mathrm{C}$ atoms is the dominant mechanism which can force locally unfavourable transfer of charges to make way for global lowering of total energy. Depletion from lone pairs of $\mathrm{N}$ would also imply reduced preference for non-coplanarity of $\mathrm{N}$ atoms with their nearest neighbours, which is indeed evident from Fig $1(\mathrm{~h})$ as it suggests lesser degree of non-coplanarity of $\mathrm{N}$ atoms in periodic supercells with non-Nn substitution. The corresponding C-N bonds in the periodic supercell are thus expected to be of order $i, 1$ which would account for their reduced length (Fig 1 1 b,d,e)), implying that they are effectively unstrained and thus energetically favourable.

Hindered $\pi$-conjugation is expected to allow the nearest neighbour anti-ferromagnetic correlation to consolidate, which is further expected to reinforce due to electron localization induced by the zigzag edges in the vicinity. In the isolated segment spin-separation is observed (Fig 2(b-c,f-i)) with all possible non-Nn substitution, whereas in the periodic supercell spin separation consolidates (Fig $2(1)$ ) only with tri-substitution at mutually N-Nn sites enclosing a common nearest neighbouring $\mathrm{C}$ atom $\left(\mathrm{C}^{*}\right.$ in $\left.\mathrm{Fig} / 3\right)$. This again reiterates that the $\mathrm{C}$ atoms would always like to maximize $\pi$-conjugation among themselves, which can be resisted effectively by the nearest neighbour antiferromagntic order only when all the three $\pi$-conjugation configurations are equally compromised, as happens with enclosing tri-substitution, or in the presence of magnetic impurity or zigzag edges. Within the region of spinseparation each $\mathrm{C}$ atom has in effect an unpaired electron centred on them (Fig]2(b-c,f-i)), indicating their partial $s p^{3}$ hybridization, which is expected to help cooperatively in allowing the local non-coplanarity preferred by the $\mathrm{N}$ atoms. However, as the $\mathrm{N}$ atoms themselves have reduced preference for non-coplanarity due to depletion from their lone pairs, an effectively planar equilibrium configuration is attained with mild systematic corrugation due to the partial $s p^{3}$ hybridization of anti-ferromagnetically correlated $\mathrm{C}$ atoms, as evident in Fig.1 $(\mathrm{g})$ for the periodic supercell with enclosing tri-substitution.

Energetics of adsorption of $\mathrm{Me}\left(\mathrm{CH}_{3}\right)$ radical on $\mathrm{C}$ atoms shown in Fig.3(a) suggests adsorption on the $\mathrm{N}$ coordinated $\mathrm{C}$ atom $\left(\mathrm{C}_{0}\right)$ due to enclosing tri-substitution to be prominently most favourable. As evident from Fig 2(b,c), mono-substitution by $\mathrm{N}$ in the isolated segment leads to spin-separation and a $\mathrm{C}-\mathrm{N}$ double bond for the majority spin. Interestingly, passivation of a $\mathrm{C}\left(\mathrm{C}^{*}\right.$ in Fig 3$)$ atom nearest to the $\mathrm{N}$ atom, exemplified here 
(a)

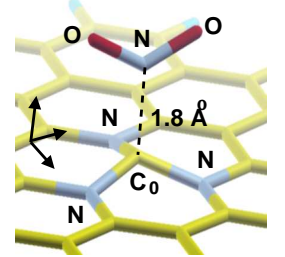

(d)

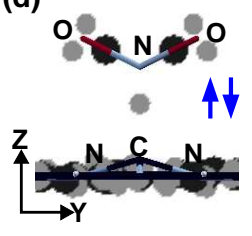

(g)

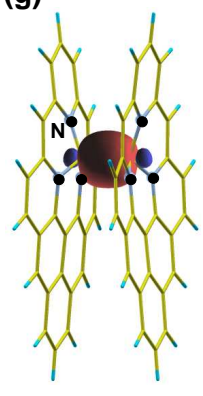

(b)

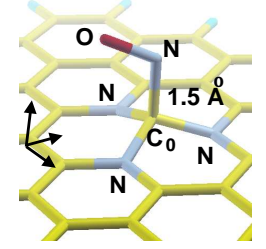

(e)

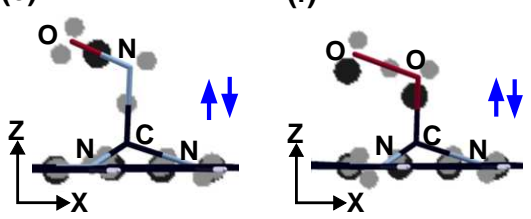

(i)

(c)

(f)
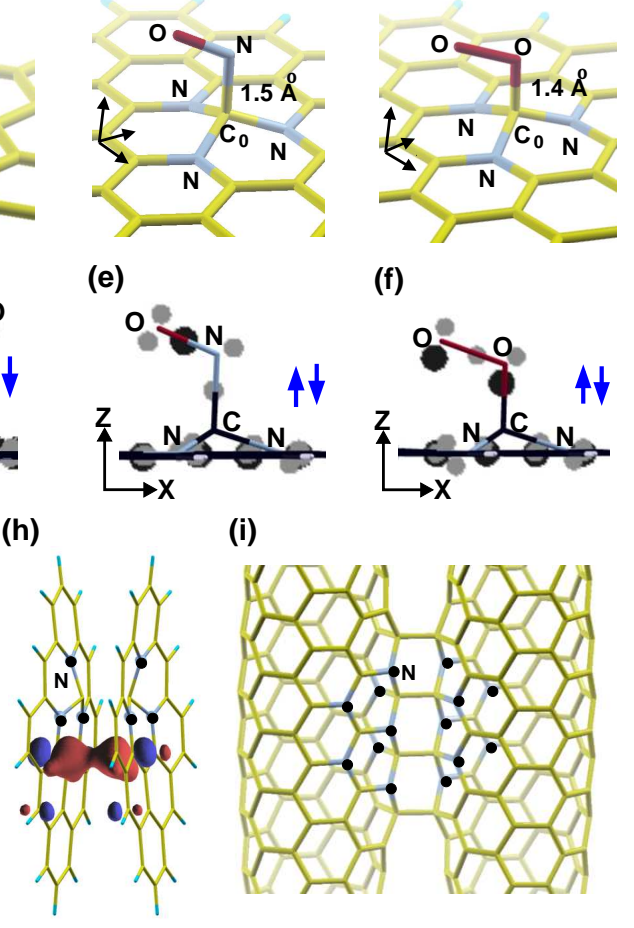

Figure 4: Adsorption of $\mathrm{NO}_{2}(\mathrm{a})$, $\mathrm{NO}(\mathrm{b})$ and $\mathrm{O}_{2}$ (c) on $\mathrm{C}_{0}$. (d-e) WCs corresponding to (a), (b) and (c) respectively, depicted by smaller(larger) gray(black) circles denoting $1(2)$ electrons per spin. (g-h) WFs representing the $\mathrm{C}_{0}-\mathrm{C}_{0}$ $\sigma$-bond (g) and an inter-segement $\pi-\pi$ bond (h). (i) Inter-CNT $\mathrm{C}_{0}-\mathrm{C}_{0}$ and adjoining $\mathrm{C}-\mathrm{C}$ bonds. $\mathrm{N}$ atoms are marked by black dots in $(\mathrm{g}-\mathrm{i})$.

through adsorption of $\mathrm{Me}$, not only restores complete $\pi$-conjugation by quenching spin-separation, but also reduces the order of the $\mathrm{C}-\mathrm{N}$ double bond (Fig $2(\mathrm{c})$ ) to 1, which restores charge neutrality of the $\mathrm{N}$ atom and relieves the tensile stresses in the adjacent graphenic $\mathrm{C}-\mathrm{C}$ bonds. In case of substitution at Nn sites, which retains $\pi$ conjugation among $\mathrm{C}$ atoms intact, passivation of any $\mathrm{C}$ atom beyond the nearest neighbourhood of $\mathrm{N}$ atoms is same as passivation of a $\mathrm{C}$ atom in an undoped segment as evident in Fig 3(a). Passivation of $\mathrm{C}$ atoms (C" in Fig 3) next to an $\mathrm{N}$ atom is more favourable on account of cooperative non-planarity of the $\mathrm{N}$ and the passivated $\mathrm{C}$ atom, although it hinders $\pi$ conjugation locally and consolidates nearest-neighbour antiferromagnetic order in the vicinity. With the experimentally observed substitution at $\mathrm{N}-\mathrm{Nn}$ sites, the $\mathrm{C}$ atom ( $\mathrm{C}^{\prime}$ in Fig 3 nearest to both the $\mathrm{N}$ atoms, neither retains charge neutrality nor completes sub-shell filling. Interestingly, both the shortcomings are restored upon passivation of C', which also makes the $\mathrm{C}$ atom next to it charge neutral, implying a higher level of activation of C' than an ordinary graphenic $\mathrm{C}$ atom, as also evident in Fig.3(a).

With enclosing tri-substitution, order of $\mathrm{C}_{0}-\mathrm{N}$ bonds (Fig, 2(h,i)) increases to around 1.5 on the average, implied by the three $\mathrm{C}-\mathrm{N}$ double bonds out of six total $\mathrm{C}-\mathrm{N}$ bonds accounting for the two spins separately. This is consistent with the shortest $\mathrm{C}-\mathrm{N}$ bond lengths observed in the periodic super-cell among the different possible $\mathrm{N}$ substitution configurations, implying high degree of mechanical stress in the adjacent C-C bonds. While strong depletion 

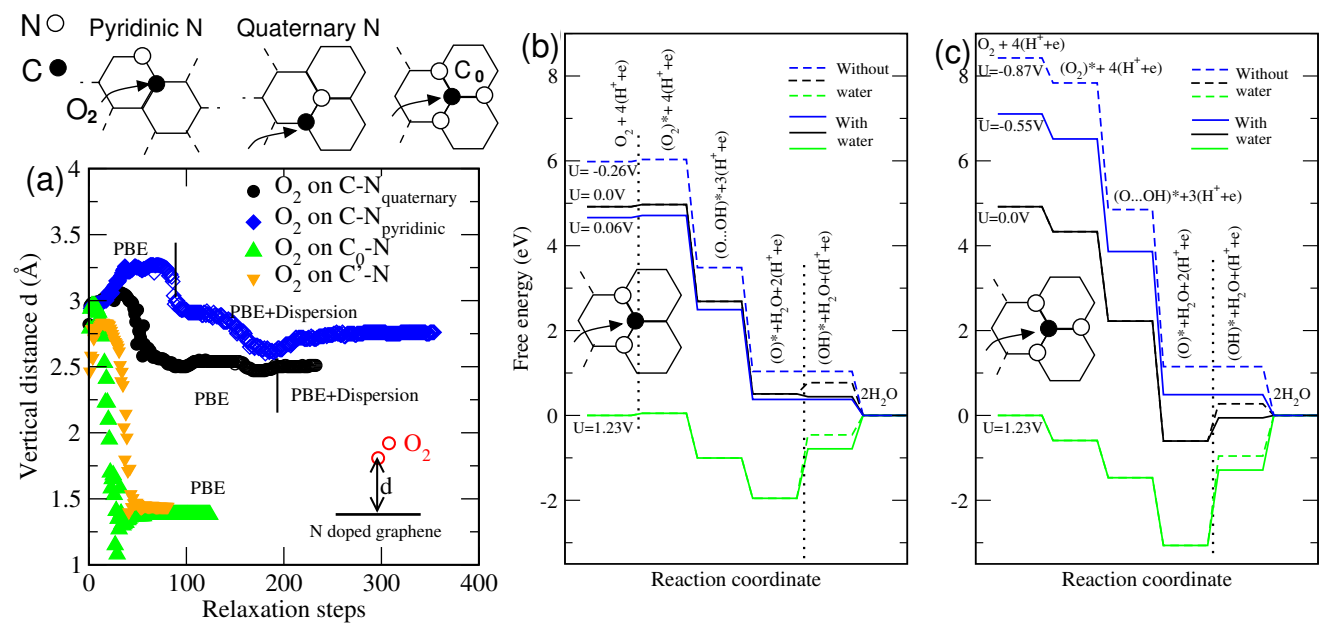

Figure 5: (a) Distance between the lower $\mathrm{O}$ of $\mathrm{O}_{2}$, and the active $\mathrm{C}$ atoms as shown in the upper panel, in course of structural relaxation using the BFGS(30) scheme. DFT + Dispersion indicates use of Grimme's approximation(31) along with PBE exchange-correlation to account for the dispersion interation. (b-c) Free energy diagram for complete reduction of the diatomic oxygen adsorbed on the active $\mathrm{C}$ atoms shown in the inset. These free diagrams are to be compared with free diagram(40) for ORR on a $\mathrm{C}$ atom next to a quaternary $\mathrm{N}$ atom, which shows an overpotential of about $0.7 \mathrm{~V}$.

from lone-pairs of $\mathrm{N}$ atoms leaves them positively charged and helps the nearest neighbour anti-ferromagnetism to consolidate, $\mathrm{C}_{0}$ would have excess charge centered on it owing to the increased order of the $\mathrm{C}_{0}-\mathrm{N}$ bonds. This excess charge on $\mathrm{C}_{0}$, being in excess to what it requires for complete sub-shell feeling, would increase total energy and should have low ionization potential. Conveniently, as evident in $(\mathrm{Fig} 22(\mathrm{j}, \mathrm{k}))$, complete $s p^{3}$ hybridization of $\mathrm{C}_{0}$, as possible upon passivation, would force all $\mathrm{C}_{0}-\mathrm{N}$ single bonds to be of order 1 , which will restore charge neutrality of of $\mathrm{N}$ atoms, besides reducing tensile stresses in the surrounding $\mathrm{C}-\mathrm{C}$ bonds and facilitating cooperative non-planarity of $\mathrm{C}_{0}$ and $\mathrm{N}$ atoms. $\mathrm{C}_{0}$ would thus be prone to passivation, and thereby, non-trivially active in terms of adsorption of radicals, as evident from the adsorption energies plotted in Fig 3(a). Interaction potential $\left(E_{(M e+G F)}-E_{M e}-E_{G F}\right)$ between Me and N doped GF plotted in Fig 3 (b) suggests consistent increase in the level of activation of $\mathrm{C}$ atoms with their increasing coordination to $\mathrm{N}$, which is directly corroborated by the adsorption energies quoted in Fig 3 (c). Spin-separation reduces significantly upon passivation of $\mathrm{C}_{0}$ in the periodic supercell primarily due to restoration of full occupancy of the lone-pair orbitals of $\mathrm{N}$.

To generalize the activation of $\mathrm{C}_{0}$ suggested by covalent adsorption of $\mathrm{Me}$, we study adsorption of two other hazardous free radicals - $\mathrm{NO}$ and $\mathrm{NO}_{2}$. As evident in Fig $4(\mathrm{a}, \mathrm{b}, \mathrm{d}, \mathrm{e})$, adsorption of $\mathrm{NO}$ and $\mathrm{NO}_{2}$ both happens through $\mathrm{C}_{0}-\mathrm{N}$ single bonds. Unlike in isolated $\mathrm{NO}$, the $\mathrm{N}$ atom in adsorbed $\mathrm{NO}$ completes sub-shell filling as well as charge neutrality(Fig, $4(\mathrm{e}))$. In adsorbed $\mathrm{NO}_{2}$, both $\mathrm{N}$ and $\mathrm{O}$ atoms are charge neutral, although the $\mathrm{N}$ atom is over coordinated on account of the two $\mathrm{N}-\mathrm{O}$ double bonds suggested by the WCs, in agreement with $\mathrm{N}-\mathrm{O}$ bond lengths of about $1.2 \AA$. Notably, an isolated $\mathrm{NO}_{2}$ molecule has resonating double and single $\mathrm{N}$-O bonds with the $\mathrm{N}(\mathrm{O})$ atom(s) positively (negatively) charged with incomplete (complete) sub-shell filling. The adsorbed configuration with two $\mathrm{N}-\mathrm{O}$ double bonds is stable since the energy of the electron at $\mathrm{N}$ in excess to what it requires to complete its sub-shell filling, is lowered due to its involvement in the $\mathrm{C}_{0}-\mathrm{N}$ covalent bond. The over coordination of $\mathrm{N}$ is reflected in the long length $(1.8 \AA)$ of the $\mathrm{C}_{0}-\mathrm{N}$ bond.

Encouraged by favourable adsorption of radicals on $\mathrm{C}_{0}$, we now probe in relation to ORR catalysis, the adsorption of $\mathrm{O}_{2}$, which is known to exist in atmosphere as diradical. Fig.5(a) unambiguously suggests spontaneous covalent adsorption of diatomic $\mathrm{O}$ on $\mathrm{C}_{0}$ and $\mathrm{C}^{\prime}$, whereas, non-nominal activation barriers appear to exist for adsorption of diatomic $\mathrm{O}$ on $\mathrm{C}$ atoms next to quaternary and pyridinic $\mathrm{N}$ atoms. Notably, pyridinic $\mathrm{N}$ atoms 
at graphene edges and quaternary $\mathrm{N}$ atoms embedded in graphene are known to adsorb oxygen through charge transfer. Ground state of $\mathrm{O}_{2}$ is a triplet where the two $\mathrm{O}$ atoms, each having an unpaired electron, are connected by an $\mathrm{O}-\mathrm{O}$ single bond. As per WC distribution, $\mathrm{O}_{2}$ appears (Fig $4(\mathrm{f})$ ) to be adsorb by $\mathrm{C}_{0}$ through a $\mathrm{O}-\mathrm{C}_{0}$ double bond.In adsorbed $\mathrm{O}_{2}$ both the $\mathrm{O}$ atoms complete sub-shell filling unlike in the $\mathrm{O}_{2}$ diradical, but both become charged in the process. The mono-coordinated outer $\mathrm{O}$ atom clearly has an extra electron (Fig $4(\mathrm{f})$ ), while the $\mathrm{O}$ atom coordinated to $\mathrm{C}_{0}$ has an electron less. Thus the $\mathrm{O}_{2}$ on the whole remain charge neutral, although their host $\mathrm{C}_{0}$ acquires an extra electron from the rest of the GN to support its over-coordination upon adsorption of $\mathrm{O}_{2}$. The $+1 \mathrm{e}$ charge of the $\mathrm{O}$ atom coordinated $\mathrm{C}_{0}$ justifies the longer length $(1.38 \AA)$ of the $\mathrm{O}_{-} \mathrm{C}_{0}$ double bond. The negatively charged outer $\mathrm{O}$ atom, although would attract cations, but may not be immediately able to donate its excess charge since such a donation would render its sub-shell filling incomplete. However, it can adsorb a radical and complete its sub-shell filling after donating its excess charge to a cation, or to the host GN itself which lost 1e to $\mathrm{C}_{0}$ upon adsorption of $\mathrm{O}_{2}$. Interestingly, the $\mathrm{O}-\mathrm{O}$ bond dissociates spontaneously upon adsorption of $\mathrm{H}$, which can be crucial for facilitation of ORR since the outer $\mathrm{O}$ atom thus acts as a free active atomic $\mathrm{O}$.

Next we study the effectiveness of the experimentally observed substitution configurations to ORR through construction of free energy diagrams(37, 38) for complete reduction of an adsorbed diatomic oxygen on C' and $\mathrm{C}_{0}$ (Fig 3). Free energy of a reduction step as a function of an applied voltage $\mathrm{U}$ is calculated as $\Delta G(U)=$ $G(0)+n e U-4 G\left(H_{2} O\right)(37)$, where $\mathrm{G}$ is the Gibbs free energy calculated as $G=E+Z P E-T S, n$ is the number of atomic $\mathrm{H}$ (proton+electron) per $\mathrm{O}_{2}$, e bring the electronic charge, and ZPE the zero point energy calculated from the vibrational frequecies. Notably, in the free energy calculations the $\mathrm{H}$ atoms are considered to be at equilibrium with molecular $\mathrm{H}_{2}$. The entropy $\mathrm{S}$ is considered only for molecules in gas phase and has been taken from standard reference(39). Free energy diagram for reduction of $\mathrm{O}_{2}$ on $\mathrm{C}$ atoms next to isolated quaternary $\mathrm{N}$ atoms suggests(40) an overpotential potential of about $0.7 \mathrm{~V}$ which is consistent with observation of ORR with $\mathrm{N}$-doped graphene in alkaline media. However, lack of adsorption of diatomic $\mathrm{O}$ on such $\mathrm{C}$ atoms can pose a limitation in terms of the availability of $\mathrm{O}$ for ORR. C' or $\mathrm{C}_{0}$ atoms on the other hand, facilitate adsorption of diatomic $\mathrm{O}$ on those $\mathrm{C}$ atoms as a diradical, but tend to bind atomic $\mathrm{O}$ strongly enough, more than $\mathrm{OH}$, to make the third reduction step, as described in Fig 5 (b,c), uphill in free energy for $\mathrm{U}=0 \mathrm{~V}$. ORR on those $\mathrm{C}$ atoms thus can only be completed through application of a large overpotential to make all the reduction steps downhill (blue lines in Fig $5(\mathrm{~b}, \mathrm{c})$ ), which is undesirably more than the equilibrium potential of $\mathrm{U}=1.23 \mathrm{~V}$. Thus the thermodynamically limiting step for ORR on $\mathrm{C}^{\prime}$ or $\mathrm{C}_{0}$ is the reduction of adsorbed $\mathrm{O}$ to $\mathrm{OH}$, whereas for the $\mathrm{C}$ atoms next to the quaternary $\mathrm{N}$ atoms the rate limiting step is the reduction of the adsorbed $\mathrm{OOH}$ or $\mathrm{OH}(40)$, which is also typically the case on metal surfaces(38). Incorporating extra stabilization(37) of adsorbed $\mathrm{OH}$ due to hydrogen bonding provided by the water molecules in the vicinity, the overpotental requirement reduces to a value marginally less than the equilibrium potential. Since increase in $\mathrm{pH}$ from zero has same effect $(G(p H)=K T \ln (10) p H(37), K$ being the Boltzmann constant) as that of lowering of an applied voltage (U), high overpotential implies ORR to be possible only in an acidic media, as has been reported(41, 42). To summarize the discussion, in one hand, next to the quaternary $\mathrm{N}$ atoms, we have the $\mathrm{C}$ atoms which appears to support ORR in acidic as well as alkaline media, but may be limited by the availability of $\mathrm{O}$ itself due to poor adsorption of diatomic $\mathrm{O}$ which dissociates only upon adsorption followed by reduction. On the hand we have the $\mathrm{C}^{\prime}$ and $\mathrm{C}_{0}$ atoms, which are coordinated to more than one $\mathrm{N}$ atoms, and ensure availability of $\mathrm{O}$ by supporting spontaneous adsorption of diatomic $\mathrm{O}$, but having high overpotential, which stipulates an acidic media. We recall here that, experimentally, $\mathrm{N}$ atoms have been observed to substitute at next nearest sites which support existence $\mathrm{C}^{\prime}$ and $\mathrm{C}_{0}$, which has been also found to be energetically favourable. Thus it appears to be a balance between the availability of $\mathrm{O}$ facilitated through adequate adsorption of $\mathrm{O}_{2}$, and the ease in their complete reduction in terms of lowering of free energy, is what would finally determine the effectiveness of $\mathrm{N}$-doped graphene as a good metal-free alternate for ORR catalysis.

In relation to mutual covalent adsorption of $\mathrm{N}$ doped $\mathrm{GNs}$, we now probe whether $\mathrm{C}_{0}$ can be passivated by a similar $\mathrm{C}$ atom in another $\mathrm{N}$ doped GN. As already shown, $s p^{3}$-hybridization of $\mathrm{C}_{0}$ due to its passivation, would preserve planarity of the rest of the GF. Steric repulsion between two such planar parallel GF segments is expected 
to make their mutual adsorption through inter-GF $\mathrm{C}_{0}-\mathrm{C}_{0}$ covalent bonds difficult due to proximity. However, additional interactions such as the dispersion interaction(D) and $\pi-\pi$ stacking interaction are expected to help in inter-GF cohesion. Strength of these interactions dependent on the nature of stacking of the segments. We study cohesion between two isolated segments starting from an initial configuration where they are 3 Aapart. Steric repulsion as well as $\pi-\pi$ stacking interaction both are expected to intensify with AA stacking. Interestingly, covalent adsorption between $\mathrm{N}$-doped GF segments due to inter-GF $\mathrm{C}_{0}-\mathrm{C}_{0}$ covalent bond (Fig $4(\mathrm{~g})$ ) is observed only with AA stacking, even without the support of dispersion interaction. Thus with AA stacking the inter-segment $\pi-\pi$ interaction, as represented by the WF shown in Fig $4(\mathrm{~h})$, is stronger than the inter-segment steric repulsion. No inter-GF adsorption with AB stacking implies sharper drop in $\pi-\pi$ interaction than that in steric repulsion compared to AA stacking, such that, the dispersion interaction(31) alone can not overcome the potential barrier induced by steric repulsion. Notably, as $\mathrm{C}_{0}$ can not be passivated by an ordinary graphenic $\mathrm{C}$ atom, $\mathrm{N}$ doping can be used to define regions of chemisorption between GF segments.

However, with reduced steric repulsion, cohesion between $\mathrm{N}$ doped CNTs appears to be easier and generic. As evident in Fig 4 (i), unlike in case of planar segments, additional inter-CNT C-C bonds are formed in the neighbourhood of the inter-CNT $\mathrm{C}_{0}-\mathrm{C}_{0}$ bonds, implying enhanced activation of $\mathrm{C}$ atoms in the neighbourhood of $\mathrm{C}_{0}$ in CNT. This is straightforward since the contractile strain caused by the $\mathrm{C}_{0}-\mathrm{N}$ bonds of higher order causes reduction in surface area, which in turn increases local curvature, and thereby, the level of $s p^{3}$-hybridization as well, leading to an enhanced level of chemical activation of $\mathrm{C}$ atoms in the neighbourhood of substitution by $\mathrm{N}$ in CNT than that in GF.

To conclude, we reveal from first principles a general scenario of non-trivial chemical activation of $\mathrm{N}$ coordinated graphenic $\mathrm{C}$ atoms upon substitution at next nearest sites as experimentally observed. Mechanical strain due to increased order of $\mathrm{C}-\mathrm{N}$ bonds and loss of charge neutrality of $\mathrm{N}$ and $\mathrm{C}$ atoms, in attempt to maximally retain $\pi$-conjugation among $\mathrm{C}$ atoms, is central to the energetics of substitution by $\mathrm{N}$, as well as the mechanisms leading to activation of $\mathrm{N}$ coordinated $\mathrm{C}$ atoms. Substitution at nearest neighbouring sites is found to be unfavourable on account of high mechanical stresses stemmed at the non-co-planarity preferred by the $\mathrm{N}$ atoms amidst strongly coplaner $\mathrm{C}$ atoms due to complete retention of $\pi$-conjugation among them. Activation is most pronounced for $\mathrm{C}$ atoms with all three nearest neighbours substituted by $\mathrm{N}$ possible upon tri-substitution at mutually N-Nn sites. Proximity to zigzag edges and curvature naturally enhances the level of activation of $\mathrm{N}$ coordinated $\mathrm{C}$ atoms. Relevant to oxygen reduction reactions (ORR), adsorption of diradical $\mathrm{O}_{2}$ on activated $\mathrm{C}$ atoms coordinated to more than one $\mathrm{N}$ atoms, renders the mono-coordinated outer $\mathrm{O}$ atom reactive, although the overpotential requirement for complete reduction of an adsorbed diatomic $\mathrm{O}$ is high due to strong binding of $\mathrm{O}$ on such $\mathrm{C}$ atoms and should allow only an acidic media. Between two adjacent GFs or CNTs, mutual covalent adsorption exclusively among the $\mathrm{N}$ coordinated $\mathrm{C}$ atoms and possibly few more $\mathrm{C}$ atoms in their close vicinity, promises a new route to robust and ordered self-assembly of GNs.

Calculations were performed partly using the central facility for high performance computing at NISER supported by the Dept. of Atomic Energy of the Govt. of India (GOI), and partly in another facility supported by a financial grant (SR/NM/NS-1026/2011) from the Dept. of Sci. and Tech. of GOI.

\section{References}

[1] Yu, D.; Nagelli, E,; Du, F.; Dai, L. Metal-free Carbon Nanomaterials Become More Active than Metal Catalysts and Last Longer. J. Phys. Chem. Lett. 2010, 1, 2165-2173.

[2] Wang, H.; Maiyalagan, T.; Wang, X. Review on Recent Progress in Nitrogen-Doped Graphene: Synthesis, Characterization, and its Potential Applications. ACS Catal. 2012, 2, 781-794.

[3] Lv, R.; Terrones, M. Towards New Graphene Materials: Doped Graphene Sheets and Nanoribbons. Mat. Let. 2012, 78, 209-218. 
[4] Gong, K.; Du, F.; Xia, Z.; Durstock, M.; Dai, L. Nitrogen-doped Carbon Nanotube Arrays with High Electrocatalytic Activity for Oxygen Reduction.Science 2009, 323, 760764.

[5] Qu, L.; Liu, Y.; Baek, JB; Dai, L. Nitrogen-doped Graphene as Efficient Metal-free Electrocatalyst for Oxygen Reduction in Fuel Cells.ACS Nano 2010, 4(3), 1321-1326.

[6] Jafri, RI; Rajalakshmi, N.; Ramaprabhu, S. Nitrogen doped Graphene Nanoplatelets as Catalyst Support for Oxygen Reduction Reaction in Proton Exchange Membrane Fuel Cell. J. Mater. Chem. 2010, 20, 7114-7117.

[7] Sheng, Z-H; Shao, L; Chen, J-J; Bao, W-J; Wang, F-B; Xia, X-H. Catalyst-free Synthesis of Nitrogen-doped Graphene via Thermal Annealing Graphite Oxide with Melamine and its Excellent Electrocatalysis. ACS Nano 2011, 5, 4350-4358.

[8] Li, Y.; Zhou, W.; Wang, H.; Xie, L.; Liang, Y.; Wei, F.; Idrobo, J-C; Pennycook, S-J; Dai, H. An Oxygen Reduction Electrocatalyst Based on Carbon Nanotube-Graphene Complexes. Nat. Nano. 2012, 7, 394-400.

[9] Sharifi, T.; Hu, G.; Jia, X.; Wagberg, T. Formation of Active Sites for Oxygen Reduction Reactions by Transformation of Nitrogen functionalities in Nitrogen-doped Carbon Nanotubes. ACS Nano 2012, 6(10), 8904-8912.

[10] Chen, S.; Bi, J.; Zhao, Y.; Yang, L.; Zhang, C.; Ma, Y; Wu, Q.; Wang, X.; Hu, Z. NitrogenDoped Carbon Nanocages as Efficient MetalFree Electrocatalysts for Oxygen Reduction Reaction. Adv. Mater. 2012, 24, $5593-5597$.

[11] Zhao, y.; Yang, L.; Chen, S.; Wang, X.; Ma, Y.; Wu, Q.; Jiang, Y.; Qian, W.; Hu, Z. Can Boron and Nitrogen Co-doping Improve Oxygen Reduction Reaction Activity of Carbon Nanotubes? J. Am. Chem. Soc. 2013, 135(4), 1201-2014.

[12] Vikkisk, M.; Kruusenberg, I.; Joost, U.; Shulga, E.; Kink, I.; Tammeveski, K. Electrocatalytic Oxygen Reduction on Nitrogen-doped Graphene in Alkaline Media. App. Catal. B: Env. 2014, 147, 369-376.

[13] Chung, H. T.; Won, J. H.; Zelenay, P. Active and Stable Carbon Nanotube/Nanoparticle Composite Electrocatalyst for Oxygen Reduction. Nat. Comm. 2013 \& 1922(1-5).

[14] Chen, P.; Xiao, T. Y.; Qian, Y. H.; Li, S. S.; Yu, S. H. A NitrogenDoped Graphene/Carbon Nanotube Nanocomposite with Synergistically Enhanced Electrochemical Activity. Adv. Mat. 2013, 25, 3192-3196.

[15] Ratso, S.; Kruusenberg, I.; Vikkisk, M.; Joost, U.; Shulga, E.; Kink, I.; Kallio, T.; Tammeveski, K. Highly Active Nitrogen-doped Few-layer Graphene/Carbon Nanotube Composite Electrocatalyst for Oxygen Reduction Reaction in Alkaline Media. CARBON 2014, 73, 361-370.

[16] Tian, G. L.; Zhao, M. Q.; Yu, D.; Kong, X. Y.; Huang, J. Q.; Zhang, Q.; Wei, F. Graphene Hybrids: NitrogenDoped Graphene/Carbon Nanotube Hybrids: In Situ Formation on Bifunctional Catalysts and Their Superior Electrocatalytic Activity for Oxygen Evolution/Reduction Reaction. Small, 2014, 10, 2113.

[17] Higgins, D.C.; Hoque, Md A.; Hassan, F.; Choi, J-Y; Kim, B.; Chen, Z. Oxygen Reduction on GrapheneCarbon Nanotube Composites Doped Sequentially with Nitrogen and Sulfur. ACS Catal., 2014, 4(8) 2734-2740. (2014).

[18] Nevidomskyy, A. H.; Gabor, C.; Payne, M. C. Chemically Active Substitutional Nitrogen Impurity in Carbon Nanotubes. Phys. Rev. Lett. 2003, 91, 105502(1-4).

[19] Wang, Z.; Jia, R.; Zheng, J.; Zhao, J.; Li, L.; Song, J.; Zhu, Z. Nitrogen-promoted Self-assembly of N-doped Carbon Nanotubes and their Intrinsic Catalysis for Oxygen Reduction in Fuel cells. ACS Nano 2011, 5(3), 1677-1684. 
[20] Hu, X.; Wu, Y.; Li, H.; Zhang, Z. Adsorption and Activation of O2 on Nitrogen-doped Carbon Nanotubes $J$. Phys. Chem. C 2010 114, 9603-9607.

[21] Kim, H.; Lee, K.; Woo, S. I.; Jung, Y. On the Mechanism of Enhanced Oxygen Reduction Reaction in Nitrogen-doped Graphene Nanoribbons. Phys. Chem. Chem. Phys. 2011, 13, 17505-17510.

[22] Zhang, L.; Xia, Z. Mechanisms of Oxygen Reduction Reaction on Nitrogen-doped Graphene for Fuel-cells. $J$. Phys. Chem. C 2011, 115(22), 11170-11176.

[23] Yu, L.; Pan, X.; Cao, X.; Hu, P.; Bao, X. Oxygen Reduction Reaction Mechanism on Nitrogen-doped Graphene: A Density Functional Theory Study J. Catal. 2011, 282, 183-190.

[24] Yan, H.,J.; Xu, B.; Shi, S., Q.; Ouyang, C., Y. First-principles Study of the Oxygen Adsorption and Dissociation on Graphene and Nitrogen doped Graphene for Li-air Batteries J. App. Phys. 2012, 112, 104316(1-5).

[25] Zhao, L.; He, R.; Taeg Rim, K.; Schiros, T.; Soo Kim, K,; Zhou, H.; Gutirrez, C.; Chockalingam, S. P.; Arguello, C., J.; Plov, L.; et al. Visualizing Individual Nitrogen Dopants in Monolayer Graphene. Science 2011, 333, 999-1003.

[26] Lv, R.; Li, Q.; Botello-Mendez, A., R.; Hayashi, T.; Wang, B.; Berkdemir, A.; Hao, Q.; Elias, A. L.; Cruz-Silva, R.; Gutierrez, H. R.; et al. Nitrogen-doped Graphene: Beyond Single Substitution and Enhanced Molecular Sensing. Sci. Rep. 2012 2, 586(1-8).

[27] Giannozzi, P.; Baroni, S.; Bonini, N.; Calandra, M.; Car, R.; Cavazzoni, C.; Ceresoli, D.; Chiarotti, G., L.; Cococcioni, M.; Dabo, I.; et al. QUANTUM ESPRESSO: A Modular and Open-source Software Project for Quantum Simulations of Materials. J. Phys.: Cond. Mat. 200921 395502(1-20)

[28] Perdew, J., P.; Burke, K.; Ernzerhof, M. Generalized Gradient Approximation made simple. Phys. Rev. Lett. 1996, 77, 3865-3868.

[29] Vanderbilt, D. Soft Self-consistent Pseudopotentials in a Generalized Eigenvalue Formalism Phys. Rev. B 1990 41(R) 7892-7895.

[30] Fletcher, R. Practical Methods of Optimization; Wiley: New York; 1987.

[31] Grimme, S. Semiempirical GGAtype Density Functional Constructed with a Longrange Dispersion Correction J. Comp. Chem. 2006, 27, 1787-1799.

[32] Wannier, G. H.; The Structure of Electronic Excitation Levels in Insulating Crystals. Phys. Rev. 1937, 52, 191-197.

[33] Kohn, W,; Construction of Wannier Functions and Applications to Energy bands. Phys. Rev. B, 1973, 7, 4388-4398.

[34] Bhattacharjee, J.; Waghmare, U., V.; Geometric phases and Wannier functions of Bloch electrons in One Dimension. Phys. Rev. B 2005, 71, 045106(1-5).

[35] Marzari, N.; Vanderbilt, D. Maximally Localized Generalized Wannier functions for Composite Energy Bands. Phys. Rev. B 1997, 56, 12847-12865.

[36] Bhattacharjee, J.; Waghmare, U., V. Localized Orbital Description of Electronic Structures of Extended Periodic Metals, Insulators, and Confined Systems: Density Functional Theory Calculations. Phys. rev. B (R) 2006, 73 121102(1-4). 
[37] Nrskov, J., K.; Rossmeisl, J.; Logadottir, A.; Lindqvist, L. Origin of the Overpotential for Oxygen Reduction at a Fuel-cell Cathode. J. Phys. Chem. B 2004 108(46), 17886-17892.

[38] Viswanathan, V.; Hansen, H., A.; Rossmeisl, J.; Nrskov, J., K. Universality in Oxygen Reduction Electrocatalysis on Metal Surfaces. ACS Catal. 2012 2(8), 1654-1660.

[39] Chase Jr., M., W. NIST-JANAF Thermochemical Tables, 4th ed. J. Phys. Chem. Ref. Data, Monograph 9, 1998.

[40] Studt, F. The Oxygen Reduction Reaction on Nitrogen-Doped Graphene. Catal. Lett. 2013 143, 58-60.

[41] Le, K., R.; Lee, K., U.; Lee, J., W.; Ahn, B., T.; Woo, S., I. Electrochemical Oxygen Reduction on Nitrogen Doped Graphene Sheets in Acid Media. Electrochem. Comm. 2010, 12, 8, 1052-1055.

[42] Zhang, L.; Niu, J.; Dai, L.; Xia, Z. Effect of Microstructure of Nitrogen-doped Graphene on Oxygen Reduction Activity in Fuel Cells Langmuir 2012, 28 7542-7550. 